\title{
Numerical Modeling and Experimental Investigation of the Nonlinear Polarization Rotation Phenomenon in Semiconductor Optical Amplifiers
}

\author{
Youssef Said and Houria Rezig \\ Sys'Com Laboratory, National Engineering School of Tunis (ENIT), B.P. 37 Le Belvedere 1002, Tunis, Tunisia \\ Correspondence should be addressed to Youssef Said, youssef.said@tunisietelecom.tn
}

Received 4 April 2011; Revised 23 August 2011; Accepted 17 September 2011

Academic Editor: Jung Huang

Copyright (C) 2011 Y. Said and H. Rezig. This is an open access article distributed under the Creative Commons Attribution License, which permits unrestricted use, distribution, and reproduction in any medium, provided the original work is properly cited.

The focus of this paper is to analyze in detail the nonlinear polarization rotation phenomenon in the Semiconductor Optical Amplifier (SOA) according to the injection conditions. To this end, we have developed a numerical model based on the coupled mode theory and the formalism of Stokes. The obtained results are in agreement with the experimental measurements that have been carried out in free space, which allows optimum control and preservation of the polarization state of the injected and collected signals.

\section{Introduction}

Semiconductor optical amplifiers (SOAs) are key enablers for optical networks. They are considered among the most promising technologies for the next generation of optical networks. They can have a significant impact on the architecture and shape the economics of future optical networks due to their attractive features, such as the compact size, the high potential of monolithically integration, and the multifunctionality combined with strong nonlinearities. They have huge potential for use in wavelength division multiplexing (WDM) and access, metropolitan, and core networks. We can achieve various optoelectronic functions for all-optical communication systems, by exploiting nonlinear effects taking place within the SOA, such as cross-gain modulation (XGM), cross-phase modulation (XPM), four-wave mixing (FWM), and cross-polarization modulation (XPolM). Therefore, SOAs can provide integrated functionality of internal switching and routing functions that are required for a feature-rich network. Space switches, wavelength converters, and wavelength selectors, which are made from SOAs, can lead to large cost reductions and improved performance in future optical network equipment.

SOAs are naturally polarization sensitive in terms of gain and effective refractive indices. The difference between the confinement factor of the transverse electric (TE) mode and that of the transverse magnetic (TM) mode results in an optical anisotropy and birefringence [1]. When two signals are injected in the SOA, an additional birefringence and gain compression affects the SOA. The two signals affect one another by producing different phase and gain compression on the TE and TM components. Consequently, this results in a rotation of the polarization state for each signal. The SOA bias current and the input signal power are the parameters that determine the magnitude of the polarization rotation.

In order to analyze the polarization rotation phenomenon in detail and to predict the SOA operational characteristics, mathematical models are required. Many theoretical and numerical models are proposed in the literature dealing with intrinsic and/or extrinsic SOA parameters [2-8]. They can be divided into two broad categories.

(i) space-resolved numerically intensive models, which take into account facet reflectivity as well as forward and backward propagating signals and amplified spontaneous emission (ASE) and offer a good fit to experimental data,

(ii) simplified analytical models with a coarser fit to experimental data but developed to facilitate conceptual understanding and performance analysis. 
In the next section, we examine the concept of the nonlinear polarization rotation phenomenon in the SOA that has been widely exploited to achieve various optoelectronic functions for all-optical telecommunications systems. In the subsequent section, we present the fundamental concept of the proposed model, which is developed to analyze the nonlinear polarization rotation. This numerical model is particularly based on the approach of the coupling mode theory, describing the coupling between the TE/TM modes and the formalism of Stokes. We follow this with details on the experimental setup, which has been conducted in the RESO Laboratory of the Brest National Engineering School in France. Then, we discuss the results obtained and compare them with the experimental ones. Finally, we conclude.

\section{Concept of the Nonlinear Polarization Rotation Phenomenon in the SOA}

The polarization of wave describes the time-varying direction of the electric field vector at a fixed point in space. It is observed along the direction of propagation by tracing out the tip of the instantaneous electric field. We can distinguish three types of wave polarization: linear, circular, and elliptical. Generally, the tip of the electric field vector traces out an ellipse and the wave is said to be elliptically polarized. Linear and circular polarizations are special cases of elliptical polarization. For the case of linearly polarized wave, the field vector is directed along a line, and its orientation is the same everywhere in space and is independent of time. However, the circularly polarized wave is characterized by a constant amplitude field vector, and the field vector orientation in space changes continuously with time so that the tip of the field vector traces out a circular locus in a plane transverse to the propagation direction.

Nonlinear polarization rotation in the SOA is caused by several effects. One of the principle causes is due to waveguide asymmetry in the device. If this later is not perfectly square, the result is that the confinement factor $(\Gamma)$ is different for TE and TM modes. Another significant type of polarization dependence present in an SOA with an asymmetric waveguide is due to the birefringence introduced to the device. This asymmetry causes two propagation constants to exist corresponding to the orthogonal TE and TM modes. The difference in effective refractive indices causes the TE and TM modes to propagate through the device at different speeds causing a phase difference to be introduced between the signals.

When two signals are injected simultaneously in the SOA, there is an induction of different phase and gain compression on the TE and TM components. Consequently, the polarization state for each signal is changed, and; thus, a polarization rotation is created. Among the parameters that determine the magnitude of the polarization rotation, we find the bias current and the input power. The nonlinear polarization rotation, which occurs in the SOA, is demonstrated to achieve very interesting applications in optical networks. However, it is exploited to assure optical gates [9-12], multiplexers [13], wavelength converters $[14,15]$, regenerators

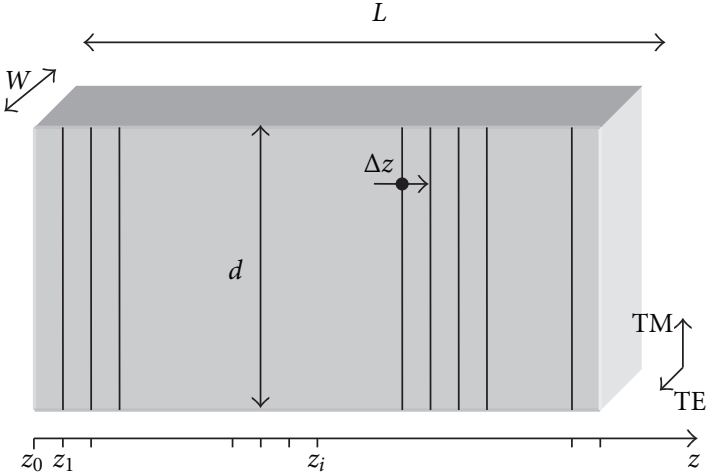

FIgURE 1: SOAs active region structure.

[15], and all-optical switching configurations [16], which are required for wavelength routing in optical networks.

To analyze the polarization rotation in an SOA structure, there are two well-known parameters, which are the azimuth $\psi$ and the ellipticity $\chi$. They are expressed as function of Stokes parameters as follows:

$$
\begin{aligned}
& \psi=\frac{1}{2} \arctan \left(\frac{s_{2}}{s_{1}}\right), \\
& \chi=\frac{1}{2} \arcsin \left(\frac{s_{3}}{\sqrt{s_{1}^{2}+s_{2}^{2}+s_{3}^{2}}}\right),
\end{aligned}
$$

where $s_{i}$ are the normalized Stokes parameters that are given by:

$$
s_{i}=\frac{S_{i}}{S_{0}}, \quad i \in\{1,2,3\},
$$

$S_{0}$ symbolizes the total intensity, $S_{1}$ is a parameter that expresses the intensity difference between the horizontal polarization and the vertical polarization, $S_{2}$ makes reference to the difference between intensities transmitted by axes $\left(45^{\circ}\right.$, $135^{\circ}$ ), and $S_{3}$ refers to the difference between intensities transmitted for the left and right circular polarizations.

\section{Fundamental Concept of the Proposed Model}

In the proposed model, we assume that the optical field is propagating in the $\mathrm{z}$-direction of the SOA structure, and it is decomposed into TE and TM components, since TE and TM are considered as the SOA quasi-eigenmodes.

The evolution of the electromagnetic field envelope in the SOA active region, which is represented in Figure 1, referring to the coupled mode equations developed in [17] taking into account the coupling between TE and TM modes, can be written as the following equations:

$$
\begin{aligned}
& \frac{\partial A_{\mathrm{TE}}}{\partial z}=\frac{g_{\mathrm{TE}}(z)}{2} \cdot A_{\mathrm{TE}}(z)+C_{1}^{\mathrm{cpl}} \cdot A_{\mathrm{TM}}(z) \cdot \exp (-j \cdot \Delta \beta \cdot z), \\
& \frac{\partial A_{\mathrm{TM}}}{\partial z}=\frac{g_{\mathrm{TM}}(z)}{2} \cdot A_{\mathrm{TM}}(z)-C_{2}^{\mathrm{cpl}} \cdot A_{\mathrm{TE}}(z) \cdot \exp (j \cdot \Delta \beta \cdot z),
\end{aligned}
$$


where

$$
\Delta \beta=\beta_{\mathrm{TM}}-\beta_{\mathrm{TE}},
$$

$\beta_{\mathrm{TE}}$ and $\beta_{\mathrm{TM}}$ symbolize the propagation constants, respectively, for TE and TM modes.

$C_{i}^{\mathrm{cpl}}$, where $i \in\{1,2\}$, denotes the coupling coefficient that is supposed to take the following form:

$$
\begin{aligned}
C_{i}^{\mathrm{cpl}}(z)= & \kappa_{i}-\kappa_{i} \cdot\left(1+\frac{\left(\left|A_{\mathrm{TE}}(z)\right|^{2}+\left|A_{\mathrm{TM}}(z)\right|^{2}\right)}{\left|E_{s}\right|^{2}}\right)^{-1} \\
& -\kappa_{i} \cdot\left(1+\frac{\left(\left|A_{\mathrm{TE}}(z)\right|^{2}+\left|A_{\mathrm{TM}}(z)\right|^{2}\right)}{\left|E_{s}\right|^{2}}\right)^{-2} \\
& -\kappa_{i} \cdot\left(1+\frac{\left(\left|A_{\mathrm{TE}}(z)\right|^{2}+\left|A_{\mathrm{TM}}(z)\right|^{2}\right)}{\left|E_{s}\right|^{2}}\right)^{-3}
\end{aligned}
$$

with " $\kappa_{i}$ " is a constant.

$g_{\mathrm{TE}}$ and $g_{\mathrm{TM}}$ represent the gain coefficients, which are supposed, in a saturated SOA, not to be constant along the amplifier length. So, they can be written as follows:

$$
\begin{gathered}
g_{\mathrm{TE}}(z)=\Gamma_{\mathrm{TE}} \cdot g_{m}(z)-\alpha_{\mathrm{TE}}, \\
g_{\mathrm{TM}}(z)=\Gamma_{\mathrm{TM}} \cdot g_{m}(z)-\alpha_{\mathrm{TM}} .
\end{gathered}
$$

$\Gamma_{\mathrm{TE}}$ and $\Gamma_{\mathrm{TM}}$ denote the confinement factors, and $\alpha_{\mathrm{TE}}$ and $\alpha_{\mathrm{TM}}$ symbolize the efficient losses, respectively, for TE and TM modes.

Additionally, in order to estimate the polarization sensitivity of a saturated amplifier, the material intensity gain coefficient $\left(g_{m}\right)$ is assumed to be saturated by the light intensity as the following equation [17]:

$$
g_{m}(z)=g_{m, 0} \cdot\left(1+\frac{\left(\left|A_{\mathrm{TE}}(z)\right|^{2}+\left|A_{\mathrm{TM}}(z)\right|^{2}\right)}{\left|E_{s}\right|^{2}}\right)^{-1} .
$$

The evolution of the electromagnetic field envelope in the active region of the SOA can also be written under the following matrix form:

$$
-j \cdot \frac{\partial A(z)}{\partial z}=M(A(z)) \cdot A(z)
$$

where:

$$
\begin{gathered}
A(z)=\left(\begin{array}{c}
A_{\mathrm{TE}}(z) \\
A_{\mathrm{TM}}(z)
\end{array}\right), \\
M(A(z))=\left(\begin{array}{ll}
m_{11} & m_{12} \\
m_{21} & m_{22}
\end{array}\right), \\
m_{11}=-\frac{j}{2} \cdot\left(\Gamma_{\mathrm{TE}} \cdot g_{m}(z)-\alpha_{\mathrm{TE}}\right), \\
m_{12}=-j \cdot C_{1}^{\mathrm{cpl}} \cdot \exp (-j \cdot \Delta \beta \cdot z), \\
m_{21}=j \cdot C_{2}^{\mathrm{cpl}} \cdot \exp (j \cdot \Delta \beta \cdot z), \\
m_{22}=-\frac{j}{2} \cdot\left(\Gamma_{\mathrm{TM}} \cdot g_{m}(z)-\alpha_{\mathrm{TM}}\right) .
\end{gathered}
$$

As the analytical solution of the differential equation set is not available, we sought to use a numerical method for calculating the electromagnetic field envelope of the SOA structure. The proposed one is based on a numerical integration approach of the differential equations in the $z$-direction. So, the initial solution of (8) at a position $z_{i+1}=z_{i}+\Delta z$, where " $i$ " is an integer, can be written as:

$$
A_{0}\left(z_{i}+\Delta z\right)=\exp \left(-j \cdot M\left(z_{i}\right) \cdot \Delta z\right) \cdot A\left(z_{i}\right) .
$$

For the fact that the matrix " $M$ " is not constant in the interval $\Delta z$, applying a correction to the initial solution is required. The correction term is expressed as.

$$
A_{c}\left(z_{i}+\Delta z\right)=j \cdot \frac{\Delta z}{2} \cdot\left[M\left(z_{i}+\Delta z\right)-M\left(z_{i}\right)\right] \cdot \overline{A_{0}},
$$

where $\overline{A_{0}}$ is the average value of $A(z)$ in the interval $\left[z_{i}, z_{i}+\right.$ $\Delta z]$. Then, the final solution is written as the following form:

$$
A\left(z_{i}+\Delta z\right)=A_{0}\left(z_{i}+\Delta z\right)+A_{c}\left(z_{i}+\Delta z\right) .
$$

For numerical implantation of the described method, the exponential term in (10) is developed as a finite summation of Taylors series terms as:

$$
\begin{aligned}
A_{0}\left(z_{i}+\Delta z\right)= & \sum_{p=0}^{q} \frac{1}{p !} \cdot\left(-j \cdot \Delta z \cdot M\left(z_{i}\right)\right)^{p} \\
& \cdot A\left(z_{i}\right)+O^{q+1}(\Delta z) .
\end{aligned}
$$

The term $O^{q+1}(\Delta z)$ denotes that the remaining error is order $(q+1)$ in $\Delta z$.

Finally, in order to reduce the calculation time, it is worthwhile to calculate the electromagnetic field envelope of SOA recursively as the following form:

$$
\begin{aligned}
\frac{1}{\mathrm{p} !} \cdot\left[-j \cdot \Delta z \cdot M\left(A\left(z_{i}\right)\right)\right]^{p} \cdot A\left(z_{i}\right) \\
=\frac{\Delta z}{p !} \cdot\left[-j \cdot M\left(A\left(z_{i}\right)\right)\right] * \frac{\Delta z^{p-1}}{(p-1) !} \\
\cdot\left[-j \cdot M\left(A\left(z_{i}\right)\right)\right]^{p-1} \cdot A\left(z_{i}\right) .
\end{aligned}
$$

In order to analyze in detail the polarization change at the SOA output with relation to its state and the value of the input power, we adopted the Stokes formalism. The reason for this choice is twofold. First, this tool has justified its robustness and simplicity to provide a very useful description of the polarization state of an electromagnetic wave. Second, it characterizes the time-averaged electric-field intensity and the distribution of polarization among three orthogonal polarization directions on the Poincaré sphere. The four Stokes parameters can be easily determined, after calculating the electromagnetic field envelope, by using the following equations:

$$
\left(\begin{array}{l}
S_{0} \\
S_{1} \\
S_{2} \\
S_{3}
\end{array}\right)=\left(\begin{array}{c}
A_{\mathrm{TE}}^{2}+A_{\mathrm{TM}}^{2} \\
A_{\mathrm{TE}}^{2}-A_{\mathrm{TM}}^{2} \\
2 A_{\mathrm{TE}} A_{\mathrm{TM}} \cdot \cos (\Delta \phi) \\
2 A_{\mathrm{TE}} A_{\mathrm{TM}} \cdot \sin (\Delta \phi)
\end{array}\right),
$$


TABLE 1: SOA parameters used in simulation.

\begin{tabular}{llc}
\hline Symbol & Description & Value \\
\hline$I_{\text {bias }}$ & Bias Current & $225 \mathrm{~mA}$ \\
$R_{1}$ & Input facet reflectivity & $5 \cdot 10^{-5}$ \\
$R_{2}$ & Output facet reflectivity & $5 \cdot 10^{-5}$ \\
$L$ & Active layer length & $500 \mu \mathrm{m}$ \\
$W$ & Active layer width & $2.5 \mu \mathrm{m}$ \\
$d$ & Active layer thickness & $0.2 \mu \mathrm{m}$ \\
$\Gamma_{\mathrm{TM}} / \Gamma_{\mathrm{TE}}$ & TM/TE optical confinement factor & 0.6 \\
$v_{g}$ & ratio & $75 \cdot 10^{6} \mathrm{~m} / \mathrm{s}$ \\
$N_{0}$ & Group velocity & $1.5 \cdot 10^{24} \mathrm{~m}^{-3}$ \\
$n_{r}$ & Carrier density at transparency & 3.7 \\
\hline
\end{tabular}

where

$$
\Delta \phi=\left(\phi_{\mathrm{TM}}-\phi_{\mathrm{TE}}\right),
$$

$\phi_{\mathrm{TE}}$ and $\phi_{\mathrm{TM}}$ designate the phase shift for TE and TM modes, respectively.

The Variation of the phase shift can be rewritten as follows:

$$
\Delta \phi=\arctan \left(\frac{S_{3}}{S_{2}}\right)
$$

The differential gain between TE and TM modes after traveling through the SOA structure, which is known as the polarization sensitivity, is given by the following equation:

$$
\begin{aligned}
\left.\Delta G\right|_{\mathrm{dB}} & =10 \log \left(\exp \left(g_{\mathrm{TE}}-g_{\mathrm{TM}}\right) \cdot L\right) \\
& =4.343\left(\left(\Gamma_{\mathrm{TE}}-\Gamma_{\mathrm{TM}}\right) \cdot g_{m}+\alpha_{\mathrm{TM}}-\alpha_{\mathrm{TE}}\right) \cdot L .
\end{aligned}
$$

The implementation of the model is made by adopting the following features: the Taylor series are being evaluated up to the twentieth order; the SOA structure is a massive type based on the InGaAsP/InP material and having the characteristics listed in Table 1.

In order to validate the results obtained by the developed model using the theoretical background described above, we have performed experiments, which are detailed in the next section.

\section{Experimental Setup}

The experimentation has been conducted in the RESO Laboratory of the Brest National Engineering School in France, by using a commercial SOA. It is a bulk and a tensile-strained structure, based on InP/GaInAsP, having the features listed in Table 2. The experimental setup, which is shown in Figure 2, was done in free space, allowing optimum control and preservation of the polarization state of the injected and collected signals. The positioning and the adjustment of optical elements in the experimentation need to be as accurate as possible in order to obtain a maximum power at the SOA output and to avoid errors in measurements. For that, we adopted three calibration steps: the first is the alignment of
TABLE 2: SOA parameters used in the experimentation.

\begin{tabular}{llc}
\hline Symbol & Description & Value \\
\hline SOA & Reference: 1550 CRI/P-SN 2106 & \\
$R_{1}$ & Input facet reflectivity & $5 \cdot 10^{-5}$ \\
$R_{2}$ & Output facet reflectivity & $5 \cdot 10^{-5}$ \\
$L$ & Active layer length & $500 \mu \mathrm{m}$ \\
$W$ & Active layer width & $2.5 \mu \mathrm{m}$ \\
$d$ & Active layer height & $0.2 \mu \mathrm{m}$ \\
$\Gamma$ & Optical confinement factor & 0.30 \\
$G_{\text {max }}$ & Maximum gain at a bias current of & $21 \mathrm{~dB}$ \\
$B_{0}$ & 225 mA and input power $P_{\text {in }}=-30 \mathrm{dBm}$ & $35 \mathrm{~nm}$ \\
$P_{\text {out }}$ sat & Bandwidth at $G_{\max }$ & $15 \mathrm{dBm}$ \\
\hline & Output saturation power at $G_{\max }$ & \\
\hline
\end{tabular}

optical beams, the second is the alignment of optical elements, and the latest is the calibration of the bench polarimeter at light running.

The SOA is placed in such a way that their TE and TM axes correspond, respectively, to the horizontal and vertical axes of the lab referential. Light emitted from the SOA was collected and collimated with a microscope objective, then passed through a quarter-wave plate (QWP) and a linear polarizer (LP) acting as an analyzer, before being recollected with a fibred collimator (FC), connected to an optical spectrum analyzer (OSA), having a resolution of $0.07 \mathrm{~nm}$. The passing axis of the linear polarizer, when set vertically, coincided with the TM axis in the sample and defined a reference direction from which the orientation $\theta$ of the fast axis of the quarter-wave plate was estimated. This orientation could be modified, as the quarter-wave plate was mounted on a rotation stage whose movements were accurately determined by a computer-controlled step motor.

All the optical elements used in the experimentation are antireflection coated around $1550 \mathrm{~nm}$, and the results discussed in this paper were systematically corrected for the spectral dependency of their transmittance.

\section{Results and Discussion}

The presence of an injected optical signal affects the carrier density and includes strong modifications of the birefringence and dichroism experienced by the signal itself in the SOA active medium. Consequently, the input signal experiences a modification of its polarization state due to the intrinsic birefringence and residual differential gain of the active region. In the linear operating regime, the output polarization remains nearly independent of the input power. However, within the saturation regime, a self-induced nonlinear rotation of polarization (SPR) takes place and depends upon input power, because of carrier density variations, which modify induced birefringence and residual differential gain. This causes fast variations of the state of polarization of the output signal, both in terms of azimuth and ellipticity.

During its crossing of the SOA cavity, the input signal will experience a modification of its polarization state due 


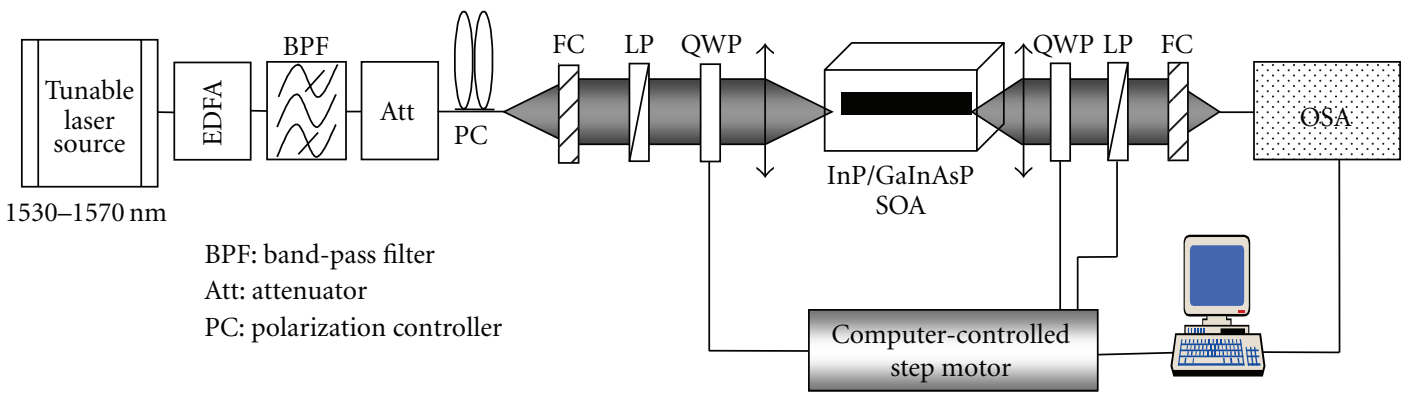

Figure 2: Experimental setup.

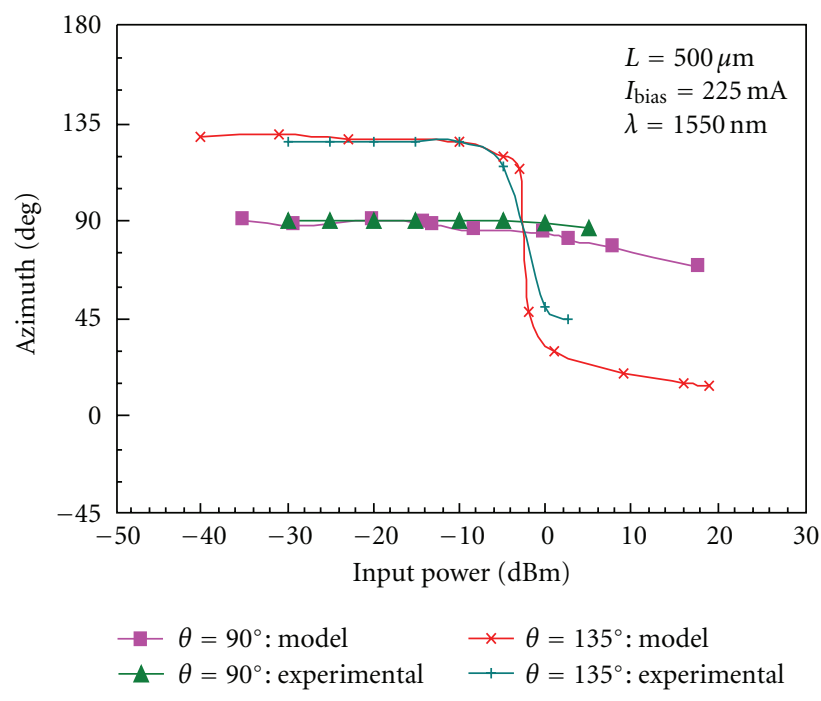

(a)

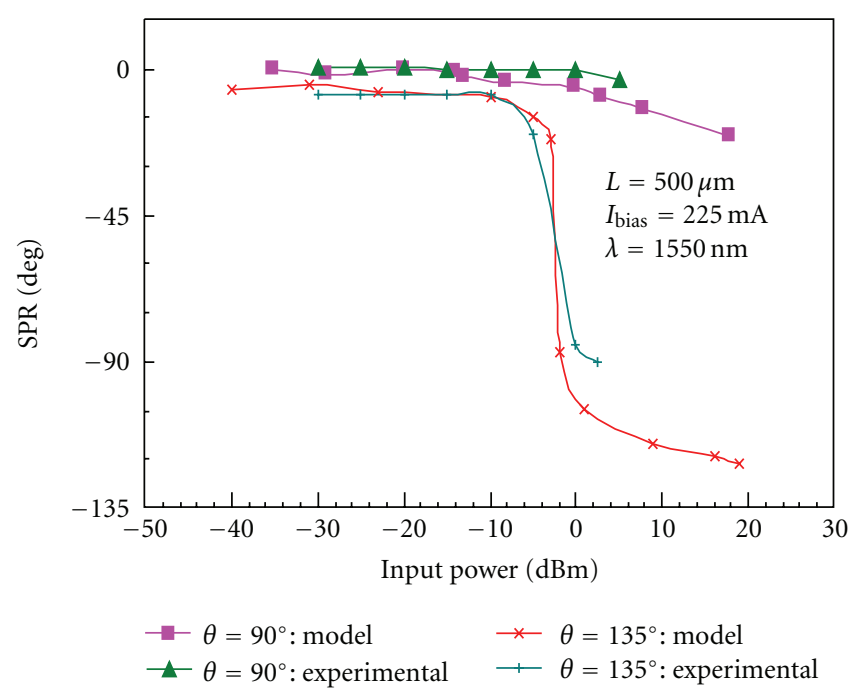

(b)

FIGURE 3: Evolution of the azimuth of the output signal and the self-induced rotation of polarization as a function of the input signal power for a bias current of $225 \mathrm{~mA}$.

to the intrinsic birefringence and residual differential gain of the active zone. Figure 3(a) depicts the evolution of the azimuth of the output state of polarization of the SOA as a function of the injected power initially injected at an angle $\theta$. This injection angle was taken equal to two values: the first is $90^{\circ}$ that corresponds to the injection along TM mode and the second is equal to $135^{\circ}$ with respect to the orientation of the eigenmodes in order to achieve identical powers for the TE and TM components. When the input signal power is low, the polarization state is almost unchanged, which corresponds to the linear operating regime. When the input power becomes high, a significant change of the polarization state is shown, particularly for an orientation angle $\theta=135^{\circ}$. This last case corresponds to the saturation regime. Moreover, we can note that simulation results are perfectly fitted to the experimental results.

The self-induced nonlinear rotation of polarization is obtained by deduction of the azimuth angle at the SOA output from the input signal injection angle $(\theta)$, which was initially taken equal to $90^{\circ}$ and $135^{\circ}$. The SPR, which is presented in Figure 3(b), is exploited to achieve various and attractive alloptical functions.
The ellipticity, which is shown in Figure 4, varies slightly for very low input signal powers. The variation of ellipticity is remarkable when there is an augmentation of the injected power that corresponds to the saturation regime of the SOA. For this case, although the simulation results are not perfectly fitted to the experimental results, we obtain a good analogy between calculation and measurements.

The phase shift variation $(\Delta \Phi)$ can be explained by those of the optically induced birefringence via the modifications of the carrier density in the SOA as the input signal power increases. According to the results presented in Figure 5(a), we can notice that $\Delta \Phi$ is almost constant when the input signal power is very low. It decreases rapidly by augmenting the injected power. This behavior is explained by the diminution of carrier density due to the stimulated emission as the input power is increased. In addition, it reflects the variation of birefringence induced by effective refractive index variations with carrier densities. Moreover, this change of $\Delta \Phi$ is significant only in the gain band, that is, when the injected input power is high, that is corresponding to the operation at the saturation regime. On the other hand, the differential gain $(\Delta G)$, which is depicted in Figure 5(b), reflects those 


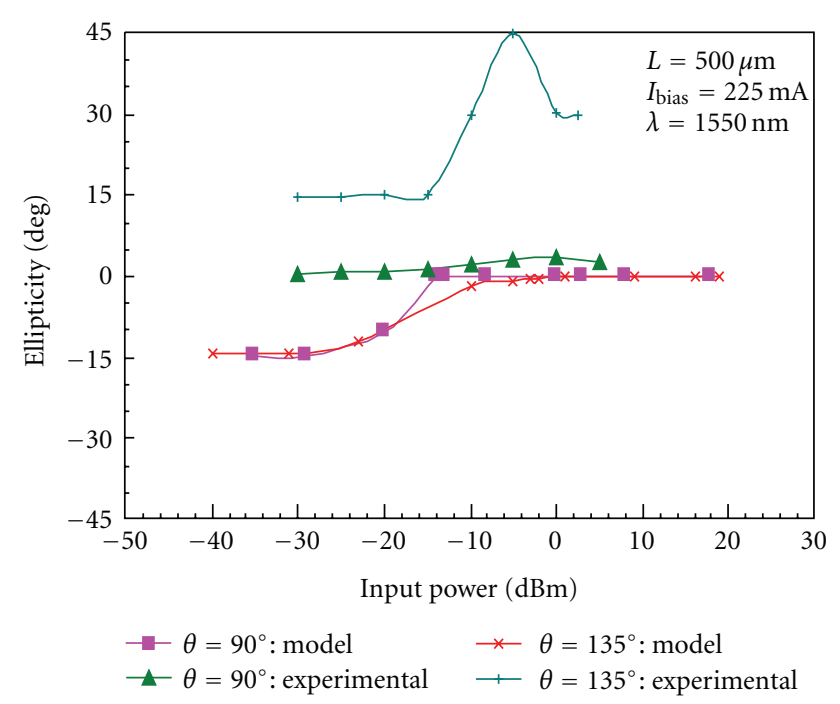

FIgURE 4: Evolution of the ellipticity of the output state of polarization of the SOA versus the input signal power for a bias current of $225 \mathrm{~mA}$.

of the dichroism induced in the SOA as the input signal power is increased. The differential gain variation, known as the polarization sensitivity, is almost constant when the input signal power is very low. It becomes significant when the input power increases. Although the simulation result of the differential gain is not perfectly fitted to the experimental one, we obtain a good analogy between them.

We can notice that the change of azimuth and ellipticity mirrors the behaviors of the refractive index and gain of the structure when the carrier density in the SOA active region is affected by the optical signal.

The difference between theoretical and experimental results presented in Figures 4 and 5 is due to many factors. In fact, there are some effects that have not been included in our model that may cause the discrepancies, which are the following.

(i) The influence of the ASE and its behavior in the presence of an injected signal: the injected signal and the ASE noise interact nonlinearly as they propagate along the SOA structure. Then, the interaction correlates different spectral components of the noise, which are the shot noise, the signal-spontaneous beat noise, and the spontaneous-spontaneous beat noise.

(ii) Dependence of the carriers' density " $N$ " on the position " $z$ ": an homogeneity of the distribution of the carriers' density is considered in $\Delta z$.

\section{Conclusion}

The key feature of the developed model is the use of the coupled mode theory and the formalism of Stokes for analyzing the nonlinear polarization rotation phenomenon in the SOA structure. The main advantage of this model is its versatility in modeling a wide range of structures and in particular, with a variety of SOA lengths, various wavelengths, and different

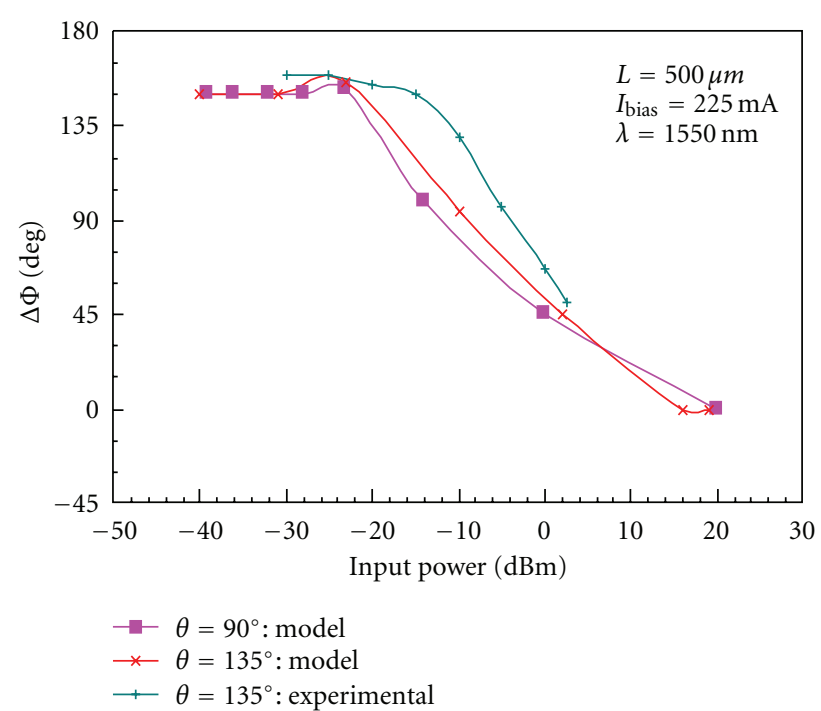

(a)

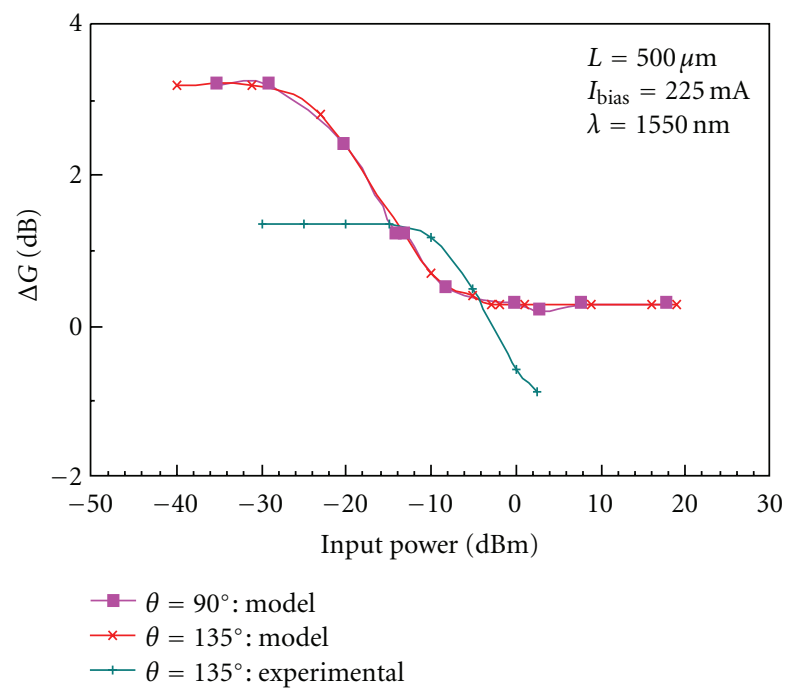

(b)

FIGURE 5: Variation of the phase shift and the differential gain of the optical signal at the SOA output as function of the injected power for a bias current of $225 \mathrm{~mA}$.

input polarization state. In order to demonstrate the performance of the developed model, we sought to examine the response of both the azimuth and the ellipticity as function of the input power signal and its polarization state. The obtained results are in agreement with the experimental measurements.

\section{Acknowledgments}

The authors would like to thank A. Sharaiha, M. Tariaki, M. Guégan, P. Morel, F. Bentivegna (RESO Laboratory, EA 3380, ENIB, Brest, France) for their fruitful discussions and cooperation in the experiments, J. Le Bihan, head of RESO 
Laboratory and A. Bouallegue, head of Sys'Com Laboratory (ENIT, Tunisia).

\section{References}

[1] L. Q. Guo and M. J. Connelly, "Signal-induced birefringence and dichroism in a tensile-strained bulk semiconductor optical amplifier and its application to wavelength conversion," Journal of Lightwave Technology, vol. 23, no. 12, pp. 4037-4045, 2005.

[2] G. Toptchiyski, S. Kindt, K. Petermann, E. Hilliger, S. Diez, and H. G. Weber, "Time-domain modeling of semiconductor optical amplifiers for OTDM applications," Journal of Lightwave Technology, vol. 17, no. 12, pp. 2577-2583, 1999.

[3] N. K. Das, Y. Yamayoshi, and H. Kawaguchi, "Analysis of basic four-wave mixing characteristics in a semiconductor optical amplifier by the finite-difference beam propagation method," IEEE Journal of Quantum Electronics, vol. 36, no. 10, pp. 11841192, 2000.

[4] R. Gutiérrez-Castrejón, L. Schares, L. Occhi, and G. Guekos, "Modeling and measurement of longitudinal gain dynamics in saturated semiconductor optical amplifiers of different length," IEEE Journal of Quantum Electronics, vol. 36, no. 12, pp. 1476-1484, 2000.

[5] M. J. Connelly, "Wideband semiconductor optical amplifier steady-state numerical model," IEEE Journal of Quantum Electronics, vol. 37, no. 3, pp. 439-447, 2001.

[6] L. Occhi, L. Schares, and G. Guekos, "Phase Modeling Based on the $\alpha$-Factor in Bulk Semiconductor Optical Amplifiers," IEEE Journal on Selected Topics in Quantum Electronics, vol. 9, no. 3, pp. 788-797, 2003.

[7] R. G. Castrejón and M. Duelk, "Uni-directional time-domain bulk SOA simulator considering carrier depletion by amplified spontaneous emission," IEEE Journal of Quantum Electronics, vol. 42, no. 6, pp. 581-588, 2006.

[8] W. Mathlouthi, P. Lemieux, M. Salsi, A. Vannucci, A. Bononi, and L. A. Rusch, "Fast and efficient dynamic WDM semiconductor optical amplifier model," Journal of Lightwave Technology, vol. 24, no. 11, pp. 4353-4365, 2006.

[9] H. Soto, D. Erasme, and G. Guekos, "5-Gb/s XOR optical gate based on cross-polarization modulation in semiconductor optical amplifiers," IEEE Photonics Technology Letters, vol. 13, no. 4, pp. 335-337, 2001.

[10] H. Soto, E. Álvarez, C. A. Díaz et al., "Design of an all-optical NOT XOR gate based on cross-polarization modulation in a semiconductor optical amplifier," Optics Communications, vol. 237, no. 1-3, pp. 121-131, 2004.

[11] J. Yang, L. Han, H. Zhang, and Y. Guo, "Function-lock strategy in OR/NOR optical logic gates based on cross-polarization modulation effect in semiconductor optical amplifier," Chinese Optics Letters, vol. 5, no. 10, pp. 566-568, 2007.

[12] H. Soto, C. A. Díaz, J. D. Topomondzo, D. Erasme, L. Schares, and G. Guekos, "All-optical AND gate implementation using cross-polarization modulation in a semiconductor optical amplifier," IEEE Photonics Technology Letters, vol. 14, no. 4, pp. 498-500, 2002.

[13] H. Soto and A. Gutiérrez, "All-optical 2-to-4 level encoder based on cross polarization modulation in a semiconductor optical amplifier utilized to develop an all-optical 2 input digital multiplexer," Optics Express, vol. 14, no. 20, pp. 9000-9005, 2006.

[14] Y. Liu, M. T. Hill, E. Tangdiongga et al., "Wavelength conversion using nonlinear polarization rotation in a single semiconductor optical amplifier," IEEE Photonics Technology Letters, vol. 15, no. 1, pp. 90-92, 2003.

[15] Y. Dong, Z. Li, C. Lu, Y. Wang, and T. H. Cheng, "3R all-optical regeneration and wavelength conversion based on cross polarization modulation effect from a single semiconductor optical amplifier," in Proceedings of the IEEE 13th Annual Meeting Lasers and Electro-Optics Society 2000 Annual Meeting (LEOS '00), vol. 1, pp. 403-404, October 2003.

[16] H. Soto, J. C. Dominguez, D. Erasme, and G. Guekos, "Demonstration of an all-optical switch using cross-polarization modulation in semiconductor optical amplifiers," Microwave and Optical Technology Letters, vol. 29, no. 3, pp. 205-208, 2001.

[17] M. Gustavsson, "Analysis of polarization independent optical amplifiers and filters based on polarization rotation in periodically asymmetric waveguides," IEEE Journal of Quantum Electronics, vol. 29, no. 4, pp. 1168-1178, 1993. 

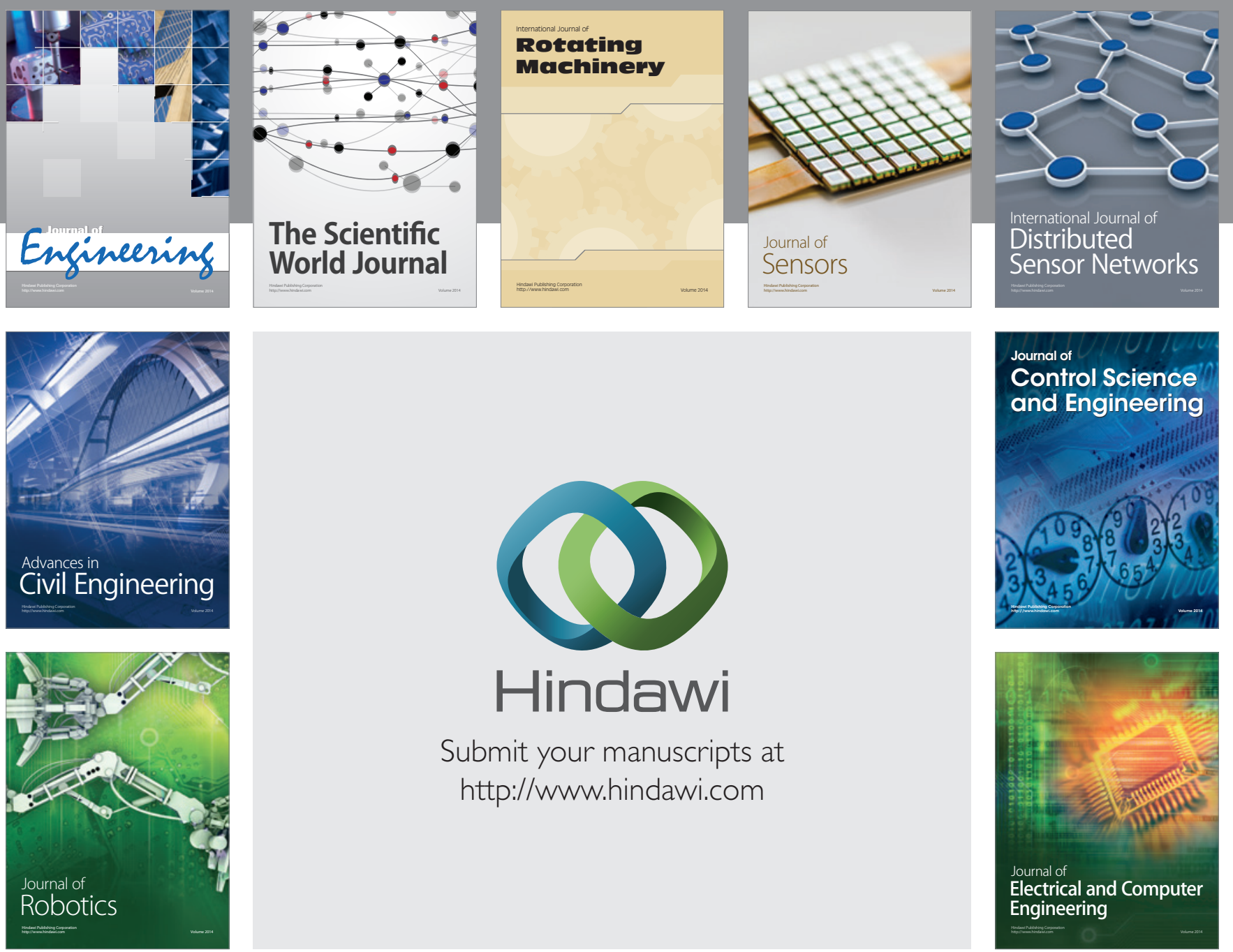

Submit your manuscripts at

http://www.hindawi.com
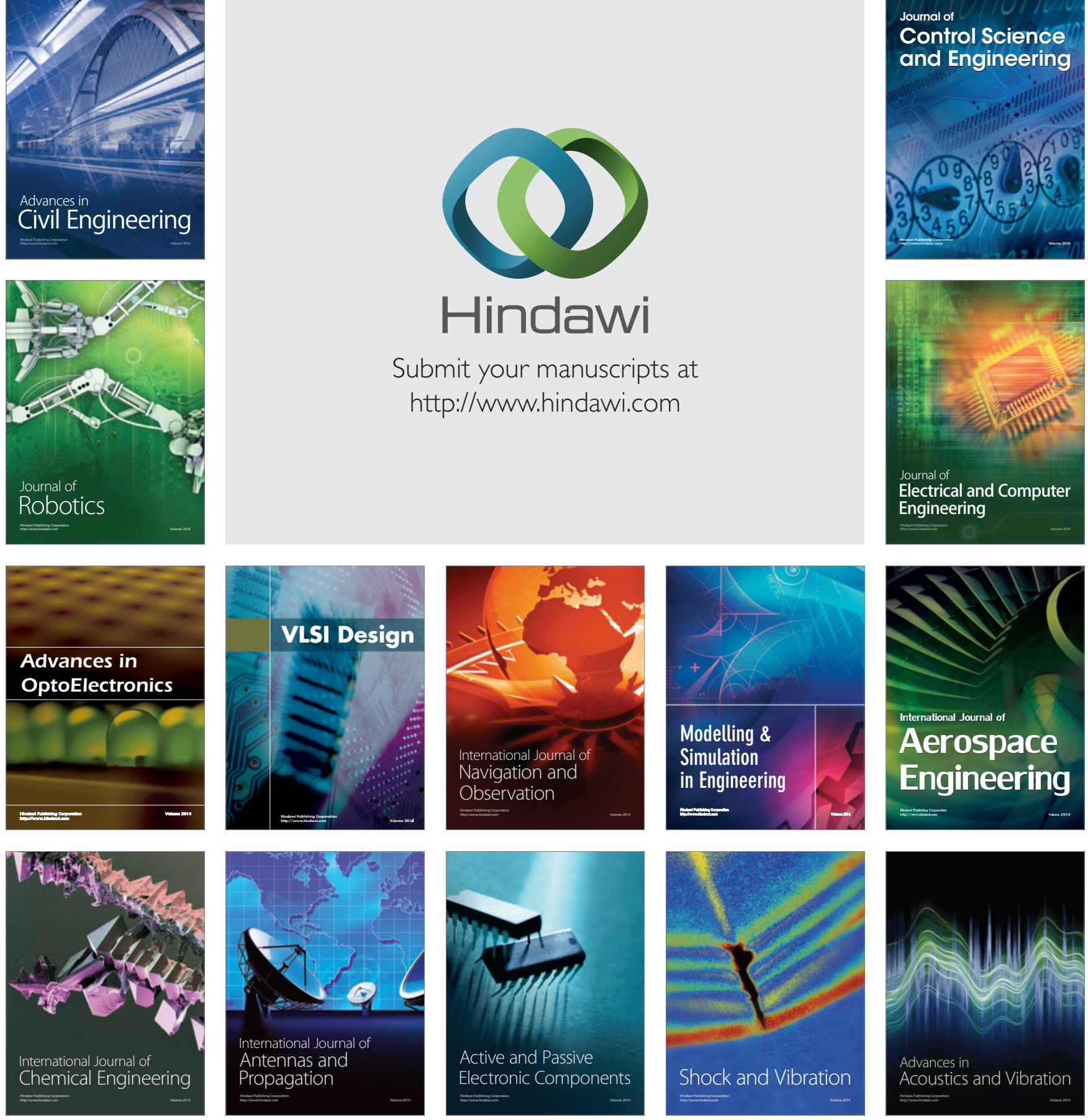